Lamarche-Gagnon et al. posit that the formation and growth of subglacial channels permits the rapid evacuation of stored methane-rich meltwater, limiting the amount of time that it is exposed to the oxygen-rich subglacial hydrological system in which bacterial oxidation occurs.

The effect on the atmosphere of the export of subglacially produced methane - and of any future growth in this methane release associated with ice-sheet retreat - will depend on several as-yet-unconstrained factors. The potential increase of methane production and export in Greenland might be limited by the area of liquid water that can form at the ice-sheet bed ${ }^{14}$. The extent of carbon-rich sediments beneath ice sheets and glaciers is also unknown, particularly in Greenland, where both sediments ${ }^{15}$ and hard beds ${ }^{16}$ have been observed.

Any increase in the proglacial methane flux from either Greenland or Antarctica will probably require a long-term expansion of subglacial hydrological systems that can efficiently evacuate stored meltwater. In Greenland, efficient subglacial drainage typically extends about 40 kilometres from the icesheet edge. Surface meltwater production in Greenland will continue to expand ${ }^{17}$, but the surface and basal topography of the ice sheet might limit the extent of efficient subglacial drainage $^{18}$, and the nature of the ice flow could limit surface-to-bed connections ${ }^{19}$.

Antarctica has extensive regions of subglacial sediments and liquid water. Increased surface meltwater and surface-to-bed connections in the future might encourage more-efficient subglacial drainage in regions where methane is produced and stored. However, any increase in subglacial methane mobilization could be mitigated if water flow is slow or if subglacial basins are large, thus allowing more-complete bacterial oxidation of methane to occur. In such scenarios, subglacial methane export might be limited to regions near the ice terminus.

Lamarche-Gagnon and colleagues' study provides an example of how our planet's icy domains can interact with the surrounding Earth system in unexpected and potentially important ways. Modelling and observational studies that characterize the ability of subglacial sediments to convert and store methane, and the ability of the subglacial hydrological system to export this methane to the atmosphere, will be key steps towards improving our knowledge of the sources and sinks of Arctic methane - and better constraining estimates of their future changes. $\square$

Lauren C. Andrews is in the Global Modeling and Assimilation Office, NASA Goddard Space Flight Center, Greenbelt, Maryland 20771, USA.

e-mail: lauren.c.andrews@nasa.gov

1. Lamarche-Gagnon, G. et al. Nature 565, 73-77 (2019).

2. Wadham, J. L. et al. Nature 488, 633 (2012).

3. Weitemeyer, K. A. \& Buffett, B. A. Glob. Planet. Change 53, 176-187 (2006).
4. Petrenko, V. V. et al. Nature 548, 443-446 (2017).

5. Christner, B. C. et al. Nature 512, 310-313 (2014)

6. Michaud, A. B. et al. Nature Geosci. 10, 582 (2017).

7. Dieser, M. et al. ISME J. 8, 2305-2316 (2014).

8. Christiansen, J. R. \& Jørgensen, C. J. Sci. Rep. 8, 16623 (2018)

9. Burns, R. et al. Sci. Rep. 8, 17118 (2018).

10.Chu, W. et al. Geophys. Res. Lett. 43, 12484-12492 (2016).

11.Hoffman, M. J., Catania, G. A., Neumann, T. A., Andrews, L. C. \& Rumrill, J. A. J. Geophys. Res. 116 F04035 (2011).

12.Cowton, T., Nienow, P., Bartholomew, I. \& Mair, D. J. Glaciol. 62, 451-466 (2016).
13. Hoffman, M. J. et al. Nature Commun. 7, 13903 (2016)

14.MacGregor, J. A. et al. J. Geophys. Res. Earth Surf. 121, 2015JF003803 (2016).

15.Walter, F., Chaput, J. \& Lüthi, M. P. Geology 42 , 487-490 (2014).

16. Harper, J. T., Humphrey, N. F., Meierbachtol, T. W., Graly, J. A. \& Fischer, U. H. J. Geophys. Res. Earth Surf. 122, 2017JF004201 (2017).

17. Leeson, A. A et al. Nature Clim. Change 5, 51-55 (2015).

18. Meierbachtol, T. W., Harper, J. \& Humphrey, N. Science 341, 777-779 (2013).

19.Poinar, K. et al. Geophys. Res. Lett. 42 2015GL063192 (2015).

\title{
Topological properties controlled by light
}

In materials called Weyl semimetals, electrons form structures that have distinct topological properties. The discovery of an ultrafast switch between two of these structures could have many practical applications. SEE LETTER P.61

\section{YOUNG-WOO SON}

$\mathrm{W}$ hen electrons flow through arrays of atoms in certain solids, they behave as quantum-mechanical particles that have extremely high speeds. Graphene - an atomically thin sheet of carbon - is an example of a material in which such behaviour occurs in two dimensions ${ }^{1}$. In the past decade, there have been worldwide efforts to study solids called Weyl semimetals, which exhibit similar intriguing properties of electrons in three dimensions ${ }^{2}$. In Weyl semimetals, electrons form structures that have peculiar topological properties, resulting in many fascinating characteristics of matter ${ }^{2}$. On-demand control of the electronic properties of a Weyl semimetal would therefore enable ultrafast manipulation of the material's properties. On page 61 , Sie et al. ${ }^{3}$ report that terahertz-frequency light can provide such control in a particular Weyl semimetal.

In the late 1920s, the physicist Paul Dirac discovered an equation that governs the behaviour of relativistic (high-speed) particles, and that combines quantum mechanics and Einstein's special theory of relativity ${ }^{4}$. Following this monumental work, the mathematician and theoretical physicist Hermann Weyl suggested a simplified version of Dirac's equation, describing massless particles - known as Weyl fermions - that have a chirality (handedness) of -1 or +1 . In a Weyl semimetal, the dynamics of low-energy electrons are governed by Weyl's equation.

The quantum state of an electron is characterized by the particle's energy, momentum and spin (intrinsic angular momentum). In a solid, these quantum states are dictated by symmetries of the material's atomic lattice.
Under time-reversal symmetry, the physical properties of a material are unchanged when the direction of time is reversed. Under inversion symmetry, the physical properties are retained when the spatial coordinates are flipped. If both of these symmetries are preserved, there are always two quantum states of electrons that have the same energy and momentum.

However, if one of these symmetries is broken, it is still possible to have quantum states of equal energy and momentum at particular points in phase space - the space of all possible energy and momentum values ${ }^{2,6}$. In the vicinity of these points, electrons are described by Weyl's equation. As a result, the elementary excitations of electrons in such solids behave as Weyl fermions, and the associated chiralities can be assigned to states near the points. By analogy with particles of opposite electric charge, states of opposite chirality can be produced in pairs, and annihilate each other when they meet.

Among the handful of Weyl semimetals that have been discovered, molybdenum ditelluride and tungsten ditelluride $\left(\mathrm{MoTe}_{2}\right.$ and $\mathrm{WTe}_{2}$, respectively) are of particular scientific inter$\mathrm{est}^{2,7}$. These compounds contain 2D structures that stack through a weak attractive force - the van der Waals interaction - to form layered 3D crystals. Depending on the stacking geometry, different crystal symmetries can be realized.

It has been known for more than four decades that, as temperature increases, $\mathrm{MoTe}_{2}$ changes from one crystal structure (orthorhombic) to another (monoclinic), whereas $\mathrm{WTe}_{2}$ does not ${ }^{8}$. Inversion symmetry is broken in the orthorhombic structure, so that the associated compounds can exhibit the Weyl-semimetal phase ${ }^{2,7}$ (Fig. 1). By contrast, 


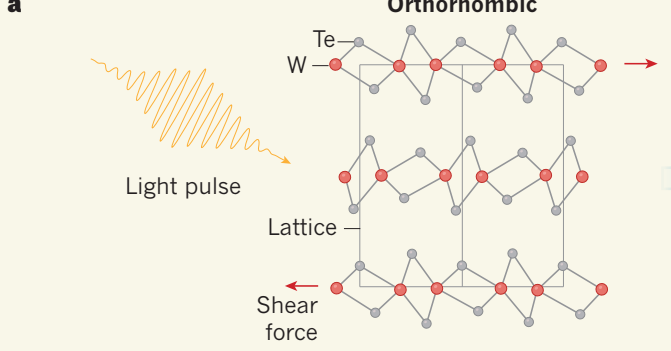

b

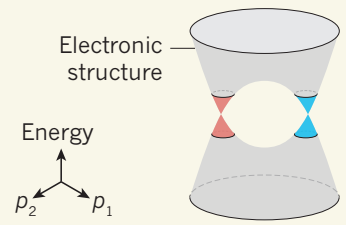

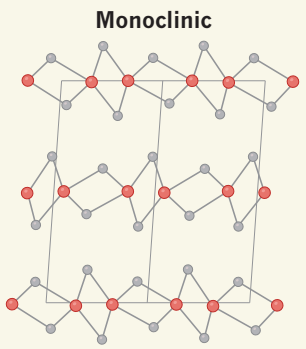

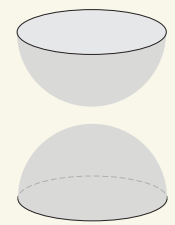

Figure 1 | Switching mechanism in a Weyl semimetal. a, Tungsten ditelluride $\left(\mathrm{WTe}_{2}\right)$ is an example of a material known as a Weyl semimetal. Sie et al. ${ }^{3}$ report a transition in $\mathrm{WTe}_{2}$ between two crystal structures: orthorhombic and monoclinic. The authors show that terahertz-frequency light pulses can transform the orthorhombic structure into the monoclinic one by altering the material's atomic lattice in a similar way to shear forces - pairs of equal and opposite forces that act on the top and bottom layers of the material. b. The dynamics of electrons in a solid can be described by a particular structure in phase space (the space of all possible energy and momentum values). Shown here are highly simplified electronic structures, in which only two components of momentum $\left(p_{1}\right.$ and $\left.p_{2}\right)$ are considered. In orthorhombic WTe $e_{2}$, electrons can behave as massless particles called Weyl fermions that have a chirality (handedness) of -1 (red) or +1 (blue). In monoclinic $\mathrm{WTe}_{2}$, these states of opposite chirality can annihilate each other.

inversion symmetry is preserved in the monoclinic structure, and the states of opposite chirality can annihilate each other. The two crystal structures have almost the same atomic lattice, except that the monoclinic one is tilted by about $4^{\circ}$ with respect to the out-of-plane direction of the orthorhombic one.

Owing to the weak attractive force between the layers of the $\mathrm{MoTe}_{2}$ and WTe ${ }_{2}$ compounds, each layer can slide easily, unlike in ordinary materials. As a result, shear forces - pairs of equal and opposite forces that act on the top and bottom layers - can deform the orthorhombic structure into the monoclinic structure, and therefore the Weyl-semimetal phase into a normal phase. Applying such forces in a mechanical way might either permanently alter the atomic lattices or be impossible. A theoretical study suggested that the crystal symmetries of these structures could instead be switched using charge doping, whereby electrons are added to or subtracted from a material ${ }^{9}$. The study indicated that this method might provide a controllable way to switch between the different topological phases.

Sie and colleagues' work is probably the first to demonstrate a dynamic transition between two crystal structures that have distinct topological phases. Previous studies have reported similar topological transitions, but these studies used static mechanical controls that cannot easily switch between the different phases $^{10,11}$. Sie et al. found that light pulses at terahertz $(\mathrm{THz})$ frequencies could cause the orthorhombic structure to become unstable by exciting electrons. This could induce the structural transition of $\mathrm{WTe}_{2}$ from orthorhombic to monoclinic, as if charge doping had been applied to the sample. The authors analysed the crystal structures using a technique known as relativistic ultrafast electron diffraction. They corroborated their measurements using a method called time-resolved second-harmonic generation, which is quite sensitive to the inversion symmetry of crystals.

All the authors' measurements clearly indicate that the crystal structure of $\mathrm{WTe}_{2}$ has inversion symmetry after the light pulses have been applied, and the switching between structures occurs at $\mathrm{THz}$ frequencies although recovery of the original structure takes much longer. Because the absence of inversion symmetry is a key characteristic of the Weyl-semimetal phase in orthorhombic $\mathrm{WTe}_{2}$, the observation of this switch of symmetries provides strong indirect evidence of the topological transition. Sie and colleagues have therefore discovered a dynamical way to control the topological properties of Weyl semimetals that could open up many applications, because the existence of Weyl fermions can substantially alter the behaviour of these materials ${ }^{2}$.

Further studies are needed to realize the full potential of the authors' switching mechanism. Because the structural transitions in $\mathrm{MoTe}_{2}$ and $\mathrm{WTe}_{2}$ are closely related to topological changes ${ }^{9}$, combined electrical and optical measurements would not only conclusively determine the topological transitions, but also provide a way to study topology-related transport phenomena in these solids ${ }^{2}$. The microscopic description of how $\mathrm{THz}$-frequency light pulses affect the electronic and structural properties of $\mathrm{WTe}_{2}$ is also required to understand the observed dynamic transitions. These endeavours and others will surely accelerate a fruitful era of topological materials and the control of these materials for applications.

Young-Woo Son is at the Korea Institute for Advanced Study, Seoul 02455, South Korea. e-mail:hand@kias.re.kr

1. Geim, A. K. \& Kim, P. Sci. Am. 298, 90-97 (2008).

2. Armitage, N. P., Mele, E. J. \& Vishwanath, A. Rev. Mod. Phys. 90, 015001 (2018).

3. Sie, E. J. et al. Nature 565, 61-66 (2019).

4. Dirac, P. A. M. Proc. R. Soc. Lond. A 117, 610-624 (1928)

5. Weyl, H. Z. Phys. 56, 330-352 (1929).

6. Herring, C. Phys. Rev. 52, 365-373 (1937)

7. Soluyanov, A. A. et al. Nature 527, 495-498 (2015).

8. Clarke, R., Marseglia, E. \& Hughes, H. P. Phil. Mag. 38, 121-126 (1978).

9. Kim, H.-J., Kang, S.-H., Hamada, I. \& Son, Y.-W. Phys. Rev. B 95, 180101(R) (2017).

10.Zeljkovic, l. et al. Nature Nanotechnol. 10, 849-853 (2015).

11.Liu, Y. et al. Nature Phys. 10, 294-299 (2014).

\section{BIOCHEMISTRY}

\section{Signalling molecule reprograms metabolism}

The signalling molecule nitric oxide protects the kidneys by reprogramming metabolism, and its levels are regulated by a two-component system in mice. These findings identify new targets for drug discovery. SEE LETTER P.96

\section{CHARLES J. LOWENSTEIN}

A cute kidney injury can lead to chronic renal failure, which causes fluid and electrolyte imbalances in the blood that require dialysis. Such injuries commonly involve ischaemia-reperfusion events, in which the blood supply to the kidney is temporarily restricted but then restored; this process generates toxic oxygen radicals that can cause renal inflammation and damage. Zhou et al. ${ }^{1}$ report on page 96 that the signalling molecule nitric oxide ${ }^{2,3}$ reprograms a metabolic pathway, and thereby limits ischaemic injury and protects renal function.

Nitric oxide is synthesized by a family of 